九州大学学術情報リポジトリ

Kyushu University Institutional Repository

\title{
アルテプラーゼ静注療法施行例の臨床的特徵と来院/ 治療開始時間の経時的変化に関する検討
}

田中，弘二

http://hdl. handle. net/2324/4060059

出版情報：Kyushu University，2019，博士（医学），課程博士 バージョン:

権利関係：○ 2019 Elsevier Inc. All rights reserved. 


\title{
Temporal Trends in Clinical Characteristics and Door-to-Needle Time in Patients Receiving Intravenous Tissue Plasminogen Activator: A Retrospective Study of 4 Hospitals in Japan
}

\author{
Koji Tanaka, MD,* Shoji Matsumoto, MD, PhD,†† Takeshi Yamada, MD, PhD, $§$ \\ Sukehisa Nagano, MD, PhD, $\uparrow$ Kei-ichiro Takase, MD, PhD, ${ }^{* *}$ \\ Taketo Hatano, MD, PhD, †+ Ryo Yamasaki, MD, PhD, ${ }^{*}$ and Jun-ichi Kira, MD, PhD*
}

\begin{abstract}
Background: Intravenous recombinant tissue plasminogen activator (rt-PA) has become a common treatment for acute ischemic stroke and has highly time-dependent benefits. We aimed to clarify temporal trends regarding the frequency and characteristics of patients receiving rt-PA and explore factors associated with doorto-needle time (DNT) in Japanese emergency hospitals. Methods: Consecutive patients who received intravenous rt-PA for acute ischemic stroke from October 2005 to December 2015 were retrospectively registered from 4 hospitals. Temporal trends in the frequency and characteristics of patients receiving rt-PA and factors associated with DNT were investigated. Results: A total of 750 patients, including 688 (420 men, median 75 years old) with out-of-hospital stroke, were registered. The frequency of patients receiving intravenous rt-PA for acute ischemic stroke continuously increased from $1.8 \%$ in 2005 to $9.5 \%$ in 2015 . The proportion of patients who were elderly or had prestroke disability increased over time, while pretreatment stroke severity declined. The DNT gradually decreased (median 105 minutes in 2005, 61 minutes in 2015). According to multivariate regression analysis with correction for multiple comparisons, activation of a code stroke system (standardized partial regression coefficient $(\beta)-.50, P<.001, \mathrm{q}<.001)$, onset-to-door time $(\beta-.15, P<.001, \mathrm{q}<.001)$, pretreatment with antithrombotic agents $(\beta .12, P<$ $.001, \mathrm{q}=.001)$, and year of treatment $(\beta .11, P=.007, \mathrm{q}=.011)$ were associated with DNT. Conclusions: Intravenous rt-PA was widely adopted in Japanese emergency hospitals. Characteristics of patients receiving intravenous rt-PA have changed over the past decade. Several factors, including the year of treatment, were associated with DNT, which has shortened over time.
\end{abstract}

Key Words: Thrombolysis—-temporal trends—door-to-needle time-stroke (c) 2019 Elsevier Inc. All rights reserved.

\footnotetext{
From the *Department of Neurology, Neurological Institute, Graduate School of Medical Sciences, Kyushu University, Fukuoka, Japan; tDepartments of Neurology, Kokura Memorial Hospital, Kitakyushu, Japan; łDepartment of Comprehensive Strokology, Fujita Health University School of Medicine, Toyoake, Japan; §Department of Neurology, Saiseikai Fukuoka General Hospital, Fukuoka, Japan; ๆDepartment of Neurology, Fukuoka City Hospital, Fukuoka, Japan; **Department of Neurology, Iizuka Hospital, Iizuka, Japan; and t+Department of Neurosurgery, Kokura Memorial Hospital, Kitakyushu, Japan.

Received May 21, 2019; revision received July 3, 2019; accepted July 21, 2019.

Financial Disclosure: This study was supported by the Japan Society for the Promotion of Science Grant-in-Aid for Scientific Research, grant number 16K10727, and Grant-in-Aid for Research Activity start-up, grant number 19K21303.

Address correspondence to Koji Tanaka, MD, Department of Neurology, Neurological Institutes, Graduate School of Medical Sciences, Kyushu University, 3-1-1 Maidashi, Higashi-ku, Fukuoka 812-8582, Japan. E-mail: tkouji@neuro.med.kyushu-u.ac.jp.

$1052-3057 / \$$ - see front matter

(c) 2019 Elsevier Inc. All rights reserved.

https:/ / doi.org/10.1016/j.jstrokecerebrovasdis.2019.104305
} 


\section{Introduction}

Intravenous recombinant tissue plasminogen activator (rt-PA) is a proven treatment for acute ischemic stroke to reduce mortality and improve functional outcomes. In Japan, intravenous rt-PA was approved in October 2005 and the percentage of patients treated with intravenous rt-PA was $5.2 \%$ in hospitals with a stroke unit and .6\% in those without. ${ }^{1}$ According to the recent Japan Standard Stroke Registry, ${ }^{2}$ intravenous rt-PA is administered to approximately $5 \%$ of all patients with acute ischemic stroke. Previous studies have demonstrated an increase in the number of patients with acute ischemic stroke who receive intravenous $\mathrm{rt}^{-} \mathrm{PA},{ }^{3-8}$ especially in patients aged $>85$ years, non-white patients, and patients with milder strokes. ${ }^{5}$ However, data on temporal trends in the clinical characteristics of patients receiving intravenous rt-PA are relatively scarce.

Because the benefit of rt-PA is highly time dependent, ${ }^{9}$ many guidelines recommend a door-to-needle time (DNT) of under 60 minutes. ${ }^{10,11}$ Previous studies showed that there were various patient-related or hospital systemrelated factors associated with the $\mathrm{DNT}^{12-15}$ and that a successful emergency medical service (EMS)-based and hospital-based performance-improvement initiative effectively shortened the DNT. ${ }^{16-18}$ However, most of these studies were conducted over a relatively short term and temporal trends in the DNT and its related factors have not been well studied.

Given this context, we aimed to clarify temporal trends regarding the frequency and clinical characteristics of patients receiving intravenous rt-PA for acute ischemic stroke since its approval and to investigate factors associated with DNTs in Japanese emergency hospitals.

\section{Materials and Methods}

\section{Study Design}

This multicenter, retrospective observational study was conducted at 4 affiliated hospitals, all of which are urban hospitals with a stroke unit. Saiseikai Fukuoka General Hospital and Iizuka Hospital are tertiary-level emergency facilities for the Fukuoka and lizuka areas, respectively, including both cities and surrounding small cities and towns. Fukuoka City Hospital and Kokura Memorial Hospital are secondary-level facilities for Fukuoka and Kitakyushu areas, respectively. Each facility has 24/7 availability of CT, MRI, and neurologists and/or neurosurgeons to provide stroke care. The cohort comprised consecutive patients who received intravenous rt-PA for acute ischemic stroke between October 1, 2005 and December 31, 2015. Trained specialized stroke physicians in each hospital performed emergency evaluations, including imaging, and made decisions about administration of rt-PA and endovascular therapy alongside thrombolysis. All patients received intravenous administration of $.6 \mathrm{mg} / \mathrm{kg}$ alteplase (the recommended dose in Japanese guidelines $^{11}$ and the approved labeling). Endovascular therapy accompanied by thrombolysis was performed for eligible patients.

Patients' clinical information was recorded in a webbased Research Electronic Data Capture database hosted at Kyushu University Hospital. ${ }^{19}$ For calculation of the annual rate of intravenous thrombolysis among acute ischemic stroke patients, the total number of patients with acute ischemic stroke admitted to each hospital during the study period was identified from the hospital discharge record of the Diagnosis Procedure Combination. The Diagnosis Procedure Combination is a mixed-case patient-classification system that includes principal diagnosis, coded according to the International Classification of Diseases and Injuries, 10th revision (ICD-10), which is linked to a hospital financing system. ${ }^{20}$ We identified patients hospitalized for acute ischemic stroke by using ICD-10 diagnosis codes related to ischemic stroke (I63.0$9)$, excluding patients with scheduled admission. This study was approved by the ethics committee of Kyushu University Hospital (29-111) and by each facility (Saiseikai Fukuoka General Hospital, Fukuoka City Hospital, lizuka Hospital, and Kokura Memorial Hospital). Written informed consent was waived because of the retrospective study design.

\section{Study Patients}

The following clinical information was systematically collected from medical records: age; sex; the presence of hypertension, diabetes mellitus, dyslipidemia, or atrial fibrillation; and pretreatment with antithrombotic agents, including antiplatelet and anticoagulant medications. Severity of stroke symptoms was assessed with the National Institutes of Health Stroke Scale (NIHSS) score, which was obtained before administration of rt-PA. Prestroke functional status was estimated with the modified Rankin Scale (mRS); prestroke disability was defined as mRS more than or equal to 3. Arrival time was classified as daytime working hours (from 8:00 AM to 4:59 PM) or night hours (from 5:00 PM to 7:59 AM), and weekdays (from Monday to Friday) or weekends (Saturday or Sunday). In hospitals with a code stroke system for earlier administration of rt-PA, whether the code stroke system was activated was assessed for patients with out-of-hospital stroke. The brain imaging modality before administration of rt-PA was classified as follows: CT only, with or without contrast enhancement (CT-based thrombolysis); MRI only (MRI-based thrombolysis); or both CT and MRI. The onset-to-door time (ODT) and the DNT were calculated from emergency medical charts for patients with out-of-hospital stroke. Symptomatic intracerebral hemorrhage was defined as CT evidence of new parenchymal intracerebral hemorrhage associated with neurological deterioration corresponding to an increase of more 
than or equal to 4 points from the baseline NIHSS score within 36 hours after treatment.

\section{Statistical Analysis}

All statistical analyses were performed with EZR version 1.37 (Saitama Medical Center, Jichi Medical University, Saitama, Japan). ${ }^{21}$ Data are expressed as medians and interquartile ranges for continuous variables and as counts and percentages for categorical variables. Clinical characteristics were compared with the Mann-Whitney's $U$ test or Cochran-Armitage or Jonckheere-Terpstra test for trend, as appropriate. Linear regression analysis was performed to investigate associations between the rates of intravenous rt-PA and the year of treatment. Multivariate regression analysis was performed to investigate factors associated with the DNT, using stepwise selection with variables entered into the model at a significance level of $\alpha=.10$ and removed at $\alpha=.10$, including all variables in the univariate analysis, in addition to the year of treatment. Because hospital characteristics vary across facilities, variables for each facility were also included. For the sensitivity analysis, DNT values were transformed with the Box-Cox transformation to better approximate a normal distribution and multivariate regression models were constructed in the manner described above. All findings were very similar; thus, for ease of interpretation we present data from the primary analysis only. For the variables included in the multivariate model, adjustment for multiple testing was applied with the Benjamini-Hochberg false discovery rate set at .05 . A $P$ value with a corresponding q value of less than .05 was considered statistically significant.

\section{Results}

Annual Rates of Intravenous rt-PA Among Patients With Acute Ischemic Stroke

A total of 750 patients were registered during the study period. According to the discharge records of the Diagnosis Procedure Combination, a total of 12,559 patients with acute ischemic stroke were admitted during the same period and were included in this analysis. Figure 1 shows annual rates of intravenous rt-PA administration among acute ischemic stroke cases. From the time of approval of rt-PA in October 2005, the rate of intravenous rt-PA use increased continuously, from $1.8 \%$ in 2005 to $9.5 \%$ in 2015 .

\section{Temporal Trends in Characteristics of Patients Receiving Intravenous rt-PA}

Of the 750 patients, 62 were excluded, 60 because of inhospital stroke and 2 because intravenous rt-PA was administered twice during the study period. A total of 688 patients (420 men; median age, 75 [66-82] years) were thus included in the final analysis. Patients' characteristics are listed in Table 1. Among patients receiving intravenous rt-PA, the percentage who were older and with prestroke disability increased over time. The proportion of patients aged more than 85 years increased from $6.0 \%$ of treated patients in $2005-2007$ to $16.0 \%$ in $2014-2015$ ( $P=$ .010 for trend). Pretreatment stroke severity declined over time and the proportion of patients with mild stroke (NIHSS score $\leq 4)$ significantly increased over time $(2.0 \%$ in 2005-2007 versus $17.7 \%$ in 2014-2015, $P<.001$ for trend). Six hundred fifty-five patients (93.5\%) were

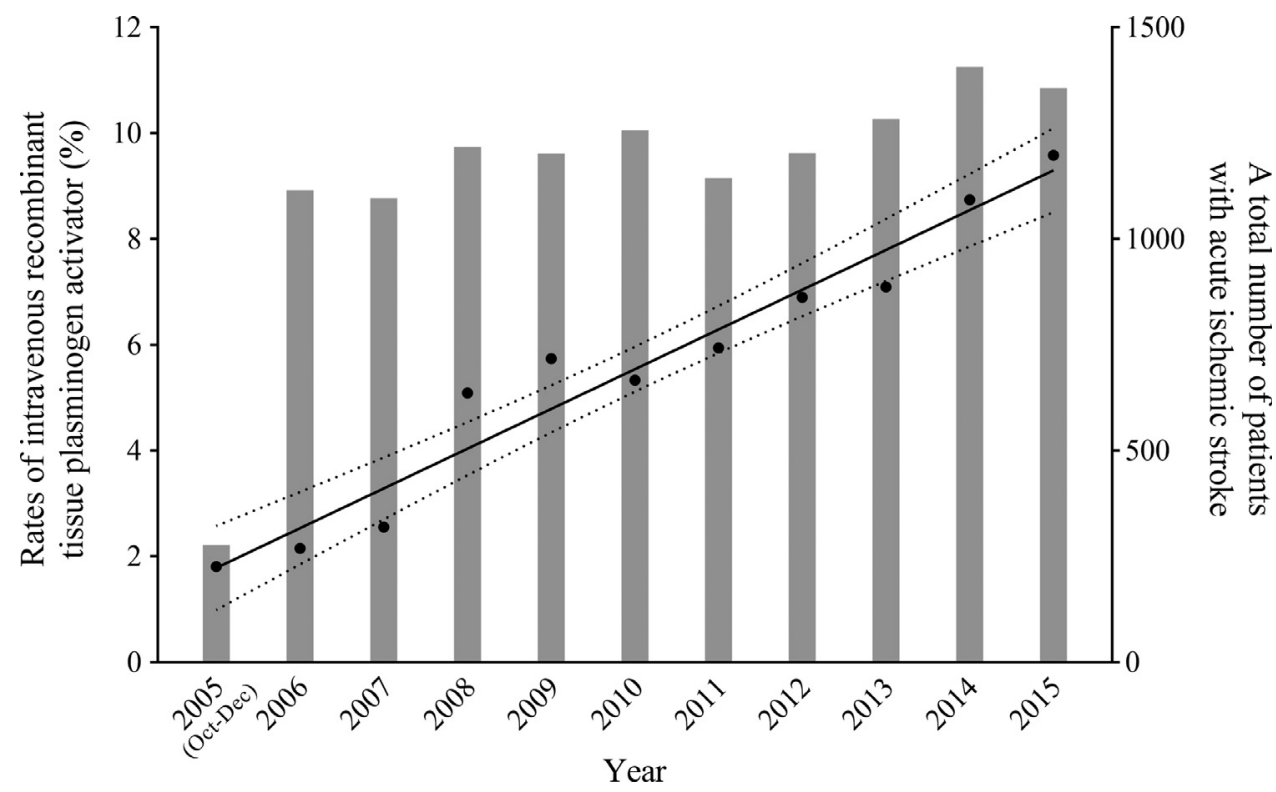

Figure 1. Annual rates of intravenous recombinant tissue plasminogen activator administration among acute ischemic stroke cases. Linear regression analysis with $\mathrm{R}^{2}=.947$ and $\mathrm{P}<.001$. 
Table 1. Temporal trends in demographics and clinical characteristics of patients receiving intravenous thrombolysis

\begin{tabular}{|c|c|c|c|c|c|c|c|}
\hline & $\begin{array}{l}\text { Total } \\
\mathrm{n}=688\end{array}$ & $\begin{array}{l}2005-2007 \\
\mathrm{n}=50\end{array}$ & $\begin{array}{l}2008-2009 \\
\mathrm{n}=124\end{array}$ & $\begin{array}{l}2010-2011 \\
\mathrm{n}=121\end{array}$ & $\begin{array}{l}2012-2013 \\
\mathrm{n}=161\end{array}$ & $\begin{array}{l}2014-2015 \\
\mathrm{n}=232\end{array}$ & $P$ value \\
\hline Sex, male* & $420(61.0)$ & $34(68.0)$ & $78(62.9)$ & $62(51.2)$ & $101(62.7)$ & $145(62.5)$ & .990 \\
\hline Age in years ${ }^{\dagger}$ & $75(66-82)$ & $71(62-78)$ & $72(64-80)$ & $78(66.5-84)$ & $75(68-81.5)$ & $76(66-84)$ & .006 \\
\hline Age $>85^{*}$ & $101(14.7)$ & $3(6.0)$ & $7(5.7)$ & $25(20.7)$ & $29(18.0)$ & $37(16.0)$ & .010 \\
\hline Prestroke disability* & $51(7.4)$ & $3(6.0)$ & $2(1.6)$ & $8(6.6)$ & $13(8.1)$ & $25(10.8)$ & .005 \\
\hline Hypertension* & $453(65.8)$ & $34(68.0)$ & $82(66.1)$ & $86(71.1)$ & $104(64.6)$ & $147(63.4)$ & .325 \\
\hline Dyslipidemia* & $185(26.9)$ & $11(22.0)$ & $35(28.2)$ & $31(25.6)$ & $42(26.1)$ & $66(28.4)$ & .547 \\
\hline Diabetes mellitus* & $157(22.8)$ & $17(34.0)$ & $20(16.1)$ & $20(16.5)$ & $45(28.0)$ & $55(23.7)$ & .510 \\
\hline Atrial fibrillation* & $349(50.7)$ & $28(56.0)$ & $67(54.0)$ & $71(58.7)$ & $73(45.3)$ & $110(47.4)$ & .052 \\
\hline Pretreatment with antithrombotics* & $266(38.7)$ & $22(44.0)$ & $50(40.3)$ & $52(43.0)$ & $62(38.5)$ & $80(34.5)$ & .106 \\
\hline Antiplatelets* & $185(26.9)$ & $15(30.0)$ & $30(24.2)$ & $32(26.4)$ & $52(32.3)$ & $56(24.1)$ & .802 \\
\hline Anticoagulants* & $103(15.0)$ & $8(16.0)$ & $25(20.2)$ & $24(19.8)$ & $18(11.2)$ & $28(12.1)$ & .027 \\
\hline Arriving in night hours* & $302(43.9)$ & $23(46.0)$ & $60(48.4)$ & $51(42.1)$ & $77(47.8)$ & $91(39.2)$ & .177 \\
\hline Arriving in weekend* & $186(27.0)$ & $11(22.0)$ & $36(29.0)$ & $26(21.5)$ & $45(28.0)$ & $68(29.3)$ & .339 \\
\hline Patients treated under code stroke* & $162(23.5)$ & $0(0)$ & $10(8.1)$ & $16(13.2)$ & $25(15.5)$ & $111(47.8)$ & $<.001$ \\
\hline Imaging before thrombolysis* & & & & & & & $<.001$ \\
\hline Both CT and MRI & $495(71.9)$ & $30(60.0)$ & $106(85.5)$ & $90(74.4)$ & $119(73.9)$ & $150(64.7)$ & \\
\hline CT-based thrombolysis & $113(16.4)$ & $19(38.0)$ & $14(11.3)$ & $7(5.8)$ & $13(8.1)$ & $60(25.9)$ & \\
\hline MRI-based thrombolysis & $80(11.6)$ & $1(2.0)$ & $4(3.2)$ & $24(19.8)$ & $29(18.0)$ & $22(9.5)$ & \\
\hline $\mathrm{ODT}^{\dagger}$ & $55(35-90)$ & $49.5(38-75)$ & $43.5(30-68)$ & $48(33-64.5)$ & $60(36.5-99)$ & $68(38.5-120)$ & $<.001$ \\
\hline $\mathrm{DNT}^{\dagger}$ & $75(59-93)$ & $69(61.5-88.5)$ & $75.5(65-93)$ & $85(70-103.5)$ & $75(61.5-93.5)$ & $65(41-90)$ & $<.001$ \\
\hline $\mathrm{DNT}<60 \mathrm{~min}^{*}$ & $193(28.1)$ & $12(24.0)$ & $23(18.5)$ & $14(11.6)$ & $39(24.2)$ & $105(45.3)$ & $<.001$ \\
\hline Preadministration NIHSS score ${ }^{\dagger}$ & $15(8-20)$ & $16.5(12-21)$ & $16(10-21)$ & $15(8-21.5)$ & $14(8-19)$ & $13(6-20)$ & $<.001$ \\
\hline NIHSS score $\leq 4 *$ & $69(10.0)$ & $1(2.0)$ & $7(5.6)$ & $5(4.1)$ & $15(9.3)$ & $41(17.7)$ & $<.001$ \\
\hline Endovascular therapy* & $110(16.0)$ & $4(8.0)$ & $2(1.6)$ & $11(9.1)$ & $32(19.9)$ & $61(26.3)$ & $<.001$ \\
\hline $\mathrm{sICH}^{*}$ & $20(2.9)$ & $1(2.0)$ & $5(4.0)$ & $6(5.0)$ & $2(1.2)$ & $6(2.6)$ & .420 \\
\hline
\end{tabular}

Abbreviations: DNT, door-to-needle time; NIHSS, National Institutes of Health Stroke Scale; ODT, onset-to-door time; sICH, symptomatic intracerebral hemorrhage.

Data are presented as $\mathrm{n}(\%)$ or median (interquartile range).

*Cochran-Armitage test for trend.

${ }^{\dagger}$ Jonckheere-Terpstra test for trend. 
ambulance-transported admissions. Two of the 4 hospitals developed and implemented a code stroke system during the study period. The system was implemented in May 2009 at Saiseikai Fukuoka General Hospital and in January 2014 at Kokura Memorial Hospital. Code stroke systems at these 2 hospitals were not identical, but both consisted of prearrival notification and activation of the entire stroke team, rapid laboratory testing, and an rt-PA toolkit. At EMS prenotification or at self-presented arrival, if the attending emergency department doctor identified patients with suspected acute ischemic stroke within the time window for intravenous rt-PA, he called the following staff immediately: an emergency room (ER) nurse, stroke physicians, a stroke unit chief nurse, and technologists (CT and MRI room, angiography room, and clinical laboratory). As laboratory tests that affect treatment decisions, point-of-care international normalized ratio and glucose measurements were performed in the ER (international normalized ratio was adopted at both facilities in 2014). A standardized rt-PA treatment kit, including rt-PA, required documents, and vascular access route set, was kept in a fixed location in the ER to allow for immediate retrieval and use. Among 209 patients who received intravenous rt-PA at these 2 hospitals after code-strokesystem implementation, $162(77.5 \%)$ were treated under the code stroke system; the remaining 47 patients were treated without code-stroke-system activation, despite implementation. Among patients admitted after May 2009, patients treated under a code stroke system had shorter DNT than other patients (median 54 [33-69] minutes versus 82 [68-103] minutes, $P<.001)$. The median DNT shortened over the study period and the proportion of patients with DNT less than 60 minutes almost doubled
(24.0\% in $2005-2007$ versus $45.3 \%$ in $2014-2015, P<.001$ for trend). The proportion of patients who received endovascular therapy alongside thrombolysis increased from $8.0 \%$ in $2005-2007$ to $26.3 \%$ in $2014-2015$ ( $P<.001$ for trend).

\section{Temporal Trends in DNT and Factors Associated With DNT}

Temporal trends in the DNT are shown in Figure 2. DNT shortened more markedly among patients treated under a code stroke system than among other patients. Multivariate regression analysis with correction for multiple comparisons (Table 2) revealed that activation of a code stroke system (standardized partial regression coefficient $(\beta)-.50, P$ $<.001)$, the ODT $(\beta-.15, P<.001)$, pretreatment with antithrombotic agents $(\beta .12, P<.001)$, and the year of treatment $(\beta .11, P=.007)$ were associated with the DNT. Patients who underwent CT only (CT-based thrombolysis, $\beta-.27, P<.001$ ) or MRI only (MRI-based thrombolysis, $\beta$ $-.13, P<.001)$ had shorter DNTs, compared with those who underwent both CT and MRI. Similar results were observed when antithrombotic agents were divided into antiplatelet and anticoagulant medications (unstandardized partial regression coefficient (B) 2.65, standard error $1.09, \beta$ $.08, \mathrm{t}=2.43, P=.015$; and B 3.55, standard error $1.33, \beta .08$, $\mathrm{t}=2.66, P=.008$, respectively).

\section{Discussion}

This study clarified temporal trends over the past decade regarding clinical characteristics of patients who received intravenous rt-PA in Japanese emergency hospitals with a stroke unit. The rate of intravenous rt-PA use

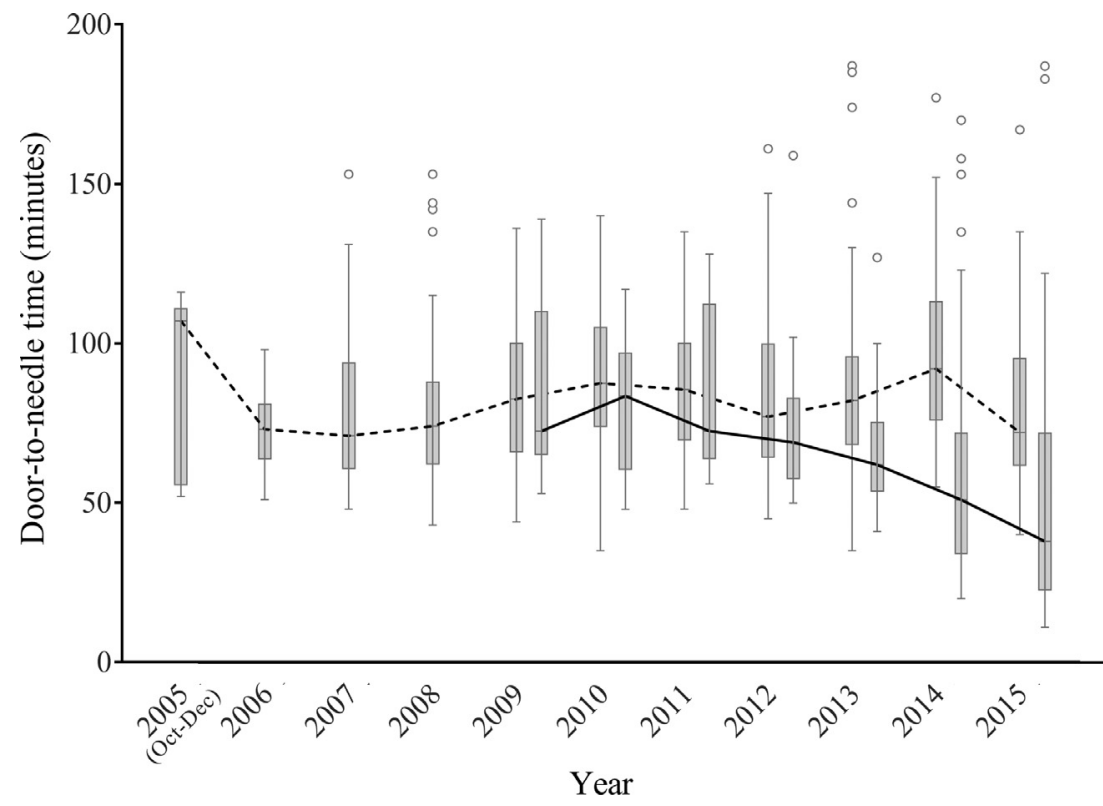

Figure 2. Temporal trends in door-to-needle times among patients treated with versus without code-stroke-system activation. Lines connect median values. The solid line represents patients treated under a code stroke system; the dotted line represents those treated without code-stroke-system activation. 
Table 2. Multiple regression analysis of door-to-needle time

\begin{tabular}{|c|c|c|c|c|c|c|}
\hline & \multicolumn{2}{|c|}{ Unstandardized } & \multirow{2}{*}{$\frac{\text { Standardized }}{\beta}$} & \multirow[b]{2}{*}{$t$ value } & \multirow[b]{2}{*}{$P$ value } & \multirow[b]{2}{*}{$q$ value } \\
\hline & B & SE & & & & \\
\hline \multicolumn{7}{|l|}{ No code stroke system (reference) } \\
\hline Code stroke system implemented but not activated & -2.75 & 2.42 & -.05 & -1.13 & .257 & .257 \\
\hline Activation of code stroke system & -17.66 & 1.77 & -.50 & -9.99 & $<.001$ & $<.001$ \\
\hline \multicolumn{7}{|l|}{ CT and MRI before thrombolysis (reference) } \\
\hline CT-based thrombolysis & -11.19 & 1.39 & -.27 & -8.04 & $<.001$ & $<.001$ \\
\hline MRI-based thrombolysis & -5.99 & 1.75 & -.13 & -3.43 & $<.001$ & .001 \\
\hline ODT, 1 min increase & -.11 & .02 & -.15 & -4.58 & $<.001$ & $<.001$ \\
\hline Pretreatment with antithrombotics & 3.70 & .99 & .12 & 3.75 & $<.001$ & .001 \\
\hline Arriving in night hours & 1.91 & .96 & .06 & 2.00 & .046 & .062 \\
\hline Sex, male & -1.90 & .98 & -.06 & -1.56 & .053 & .064 \\
\hline Year of treatment, 1 year past from 2005 & 1.18 & .44 & .11 & -2.72 & .007 & .011 \\
\hline Adjusted $R^{2}$ & .331 & & & & & \\
\hline
\end{tabular}

Abbreviations: ODT, onset-to-door time; SE, standard error. The model was adjusted for the facility variable.

for acute ischemic stroke continued to increase during the 10 years after its approval in October 2005. In the United States, rt-PA was approved in 1996. However, the use of rt-PA began to increase only in the early 2000s at high-volume academic hospitals and in 2006 at community hospitals. ${ }^{4,7}$ By contrast, in Sweden the frequency of patients receiving intravenous rt-PA rapidly increased after its approval in 2003. ${ }^{3}$ A similar rapid increase was observed in the present study. International evidence of the benefits of rt-PA reported during the period before drug approval in Japan might explain the rapid increase in rt-PA use after its approval in 2005.

This study showed a temporal trend of increased rt-PA use for older patients and those with mild stroke. These findings corroborate those of a previous report from hospitals using the Get With the Guidelines-Stroke program from 2003 to $2011 .^{5}$ Moreover, in the October 2012 revision of the Japan Stroke Society's guidelines for the intravenous application of $\mathrm{rt}-\mathrm{PA}^{11}$ the target population criteria were expanded; several exclusion criteria were removed (eg, ischemic stroke within 3 months, mild stroke deficits, and rapidly improving stroke symptoms) and the upper age limit for intravenous rt-PA administration in selected patients was revised from 75 to 81 years. These revisions of the guidelines might have led to increased administration of rt-PA in elderly patients and patients with mild stroke. Additionally, this study showed an increase in rt-PA use in patients with prestroke disability. Prestroke disability is more common among stroke patients aged more than 75 years ${ }^{22}$ and has been associated with the decision not to offer rt-PA. ${ }^{23}$ However, rt-PA is beneficial even in patients with severe prestroke disability. ${ }^{24}$ Given the aging of the population, vascular neurologists and neurosurgeons will more frequently need to assess the indications for rt-PA in acute stroke patients with prestroke disability.
The median DNT gradually decreased over the study period. Despite the decreasing trend in DNT, later year of treatment was associated with longer DNT in multivariate analysis. There are 2 possible reasons for this finding. First, intravenous rt-PA within 4.5 hours of onset was approved in August 2012; guidelines were revised in October $2012^{11}$ to allow even patients with long DNT to receive rt-PA. Second, some patient characteristics changed over time but were not selected in stepwise selection. Previous reports showed that DNT was longer among older patients and those with lower NIHSS score. ${ }^{12,15,25}$ The proportion of patients with these characteristics increased during the study period. Our results support those of a previous report that found that DNT did not decrease much without specific interventions. ${ }^{26}$

Activation of a code stroke system had the most significant impact on shortening the DNT, a finding in accordance with previous reports showing that an EMS- and hospital-based performance-improvement initiative including a code stroke system was effective for shortening the DNT. ${ }^{16-18,27-29}$ In the present study, the code stroke system was activated for $77.5 \%$ of patients receiving rt-PA in the hospitals where it was implemented. This rate was comparable with that in the previous study from Iglesias Mohedano et $\mathrm{al}^{29}$ in which a prehospital code stroke was activated for $88.3 \%$ of patients receiving rt-PA. In the present retrospective study, some hospitals had independently implemented code stroke systems while others had not. This study allowed investigation of the effect of implementation and activation of a code stroke system on DNT by comparing hospitals with and without implementation of a code stroke system during the same period.

Pretreatment with antithrombotic agents, both antiplatelet and anticoagulant medications, was associated with longer DNTs, as shown in a previous study. ${ }^{15}$ 
The most recent guidelines from the American Heart Association recommend intravenous rt-PA for patients taking antiplatelet agents or warfarin who have an international normalized ratio less than or equal to $1.7^{10}$; Japanese guidelines recommend that rt-PA should be carefully administered in selected patients taking those medications. ${ }^{11}$ Because uncertainty about anticoagulation status delays $\mathrm{DNT}^{14}$ screening for medical contraindications for rt-PA, measuring prothrombin time in suitable patients, and obtaining informed consent for the increased risk of hemorrhage might prolong the DNT in patients receiving antithrombotic agents.

The age and sex distributions, preadministration imaging modality, and stroke severity of patients in this study were similar to those of the Stroke Acute Management with Urgent Risk-Factor Assessment and Improvement rt-PA registry. ${ }^{30}$ Although the sample size was small, the population of this study reflects at least in part those in current clinical practice in Japanese emergency hospitals. Some of the factors revealed in this study are modifiable and argue for the introduction of medical resources for the timely administration of rt-PA. One recent study reported that EMS with prehospital notification resulted in shorter DNT and ODT. A shorter onset-to-treatment time was associated with better neurological outcomes. ${ }^{31}$ Although the 3-month mRS was not investigated in the present study, the shortening trend in DNT, especially that derived from a code stroke system, may have contributed to improved stroke outcomes.

Our study has several limitations. First, the study has a retrospective design with a small number of patients from limited facilities, which might have created selection bias. Second, because of the retrospective study design, not all factors that have been reported to be associated with the DNT were included (eg, fluctuating neurological deficits, incorrect triage, and other logistic factors). Third, we used ICD-10 diagnosis codes to determine the population with acute ischemic stroke, which was not validated. Moreover, details of patients' background characteristics, including changes in the proportion of patients over 85 years of age or with mild stroke in the overall population of acute ischemic stroke patients, remain unclear. Finally, associations between temporal trends in patients' clinical characteristics and the DNT and clinical outcomes were not investigated.

\section{Conclusions}

Since 2005, intravenous rt-PA has become widely adopted for the treatment of acute ischemic stroke in Japanese emergency hospitals with a stroke unit. The administration of rt-PA to older patients, those with prestroke disability, and those with mild stroke has increased. Alongside factors described previously, the year of treatment was associated with DNT, which has shortened over time.

\section{Acknowledgments}

The authors thank the Center for Clinical and Translational Research, Kyushu University, for the maintenance and management of the database and Dr Chie Kikutake, Medical Institutes of Bioregulation, Kyushu University, for advice on statistical analyses. We also thank Rebecca Tollefson, DVM, from Edanz Group (www.edanzediting.com/ac) for editing a draft of this manuscript.

\section{Conflicts of Interest}

The authors have no financial conflicts of interest.

\section{References}

1. Sato S, Uehara T, Toyoda K, et al. Impact of the approval of intravenous recombinant tissue plasminogen activator therapy on the processes of acute stroke management in Japan: the Stroke Unit Multicenter Observational (SUMO) Study. Stroke 2009;40:30-34.

2. Kobayashi S. Stroke databank 2015. Tokyo: Nakayamashoten; 2015 [in Japanese].

3. Eriksson M, Jonsson F, Appelros P, et al. Dissemination of thrombolysis for acute ischemic stroke across a nation: experiences from the Swedish stroke register, 2003 to 2008. Stroke 2010;41:1115-1122.

4. Adeoye $\mathrm{O}$, Hornung $\mathrm{R}$, Khatri $\mathrm{P}$, et al. Recombinant tissue-type plasminogen activator use for ischemic stroke in the United States: a doubling of treatment rates over the course of 5 years. Stroke 2011;42:1952-1955.

5. Schwamm LH, Ali SF, Reeves MJ, et al. Temporal trends in patient characteristics and treatment with intravenous thrombolysis among acute ischemic stroke patients at Get With The Guidelines-Stroke hospitals. Circ Cardiovasc Qual Outcomes 2013;6:543-549.

6. Hsieh $\mathrm{CY}$, Chen $\mathrm{CH}$, Chen $\mathrm{YC}$, et al. National survey of thrombolytic therapy for acute ischemic stroke in Taiwan 2003-2010. J Stroke Cerebrovasc Dis 2013;22:e620-e627.

7. Domino JS, Baek J, Meurer WJ, et al. Emerging temporal trends in tissue plasminogen activator use: results from the BASIC project. Neurology 2016;87:2184-2191.

8. Khurana D, Das B, Kumar A, et al. Temporal trends in intravenous thrombolysis in acute ischemic stroke: experience from a tertiary care center in India. J Stroke Cerebrovasc Dis 2017;26:1266-1273.

9. Saver JL, Fonarow GC, Smith EE, et al. Time to treatment with intravenous tissue plasminogen activator and outcome from acute ischemic stroke. JAMA 2013;309:24802488.

10. Powers WJ, Rabinstein AA, Ackerson T, et al. 2018 Guidelines for the early management of patients with acute ischemic stroke: a guideline for healthcare professionals from the American Heart Association/American Stroke Association. Stroke 2018;49:e46-e110.

11. Minematsu K, Toyoda K, Hirano T, et al. Guidelines for the intravenous application of recombinant tissue-type plasminogen activator (alteplase), the second edition, October 2012: a guideline from the Japan Stroke Society. J Stroke Cerebrovasc Dis 2013;22:571-600.

12. Fonarow GC, Smith EE, Saver JL, et al. Timeliness of tissue-type plasminogen activator therapy in acute ischemic stroke: patient characteristics, hospital factors, and outcomes associated with door-to-needle times within 60 minutes. Circulation 2011;123:750-758. 
13. Mikulík R, Kadlecová P, Czlonkowska A, et al. Factors influencing in-hospital delay in treatment with intravenous thrombolysis. Stroke 2012;43:1578-1583.

14. Van Schaik SM, Scott S, de Lau LM, et al. Short door-toneedle times in acute ischemic stroke and prospective identification of its delaying factors. Cerebrovasc Dis Extra 2015;5:75-83.

15. Kamal N, Sheng S, Xian Y, et al. Delays in door-to-needle times and their impact on treatment time and outcomes in get with the guidelines-stroke. Stroke 2017;48:946-954.

16. Meretoja A, Strbian D, Mustanoja S, et al. Reducing in-hospital delay to 20 minutes in stroke thrombolysis. Neurology 2012;79:306-313.

17. Fonarow GC, Zhao X, Smith EE, et al. Door-to-needle times for tissue plasminogen activator administration and clinical outcomes in acute ischemic stroke before and after a quality improvement initiative. JAMA 2014;311:1632-1640.

18. Hsieh FI, Jeng JS, Chern CM, et al. Quality improvement in acute ischemic stroke care in Taiwan: the breakthrough collaborative in stroke. PLoS ONE 2016;11:e0160426.

19. Harris PA, Taylor R, Thielke R, et al. Research electronic data capture (REDcap): a metadata-driven methodology and workflow process for providing translational research informatics support. J Biomed Inform 2009;42:377-381.

20. Yasunaga H, Ide H, Imamura $T$, et al. Impact of the Japanese Diagnosis Procedure Combination-based Payment System on cardiovascular medicine-related costs. Int Heart J 2005;46:855-866.

21. Kanda Y. Investigation of the freely-available easy-to-use software 'EZR' for medical statistics. Bone Marrow Transplant 2013;48:452-458.

22. Sharma JC, Fletcher S, Vassallo M. Strokes in the elderly-higher acute and 3-month mortality-an explanation. Cerebrovasc Dis 1999;9:2-9.
23. Cappellari M, Bosco M, Forlivesi S, et al. Reasons for exclusion from intravenous thrombolysis in stroke patients admitted to the Stroke Unit. J Thromb Thrombolysis 2016;42:593-599.

24. Karlinski M, Kobayashi A, Czlonkowska A, et al. Role of preexisting disability in patients treated with intravenous thrombolysis for ischemic stroke. Stroke 2014;45: 770-775.

25. Rostanski SK, Shahn Z, Elkind MSV, et al. Door-to-needle delays in minor stroke: a causal inference approach. Stroke 2017;48:1980-1982.

26. Strbian D, Ahmed N, Wahlgren N, et al. Trends in doorto-thrombolysis time in the safe implementation of stroke thrombolysis registry: effect of center volume and duration of registry membership. Stroke 2015;46:1275-1280.

27. Chen CH, Tang SC, Tsai LK, et al. Stroke code improves intravenous thrombolysis administration in acute ischemic stroke. PLoS ONE 2014;9:e104862.

28. Busby L, Owada K, Dhungana S, et al. CODE FAST: a quality improvement initiative to reduce door-to-needle times. J Neurointerv Surg 2016;8:661-664.

29. Iglesias Mohedano AM, García Pastor A, García Arratibel A, et al. Factors associated with in-hospital delays in treating acute stroke with intravenous thrombolysis in a tertiary centre. Neurologia 2016;31:452-458.

30. Toyoda K, Koga M, Naganuma M, et al. Routine use of intravenous low-dose recombinant tissue plasminogen activator in Japanese patients: general outcomes and prognostic factors from the SAMURAI register. Stroke 2009;40:3591-3595.

31. Zhang S, Zhang J, Zhang M, et al. Prehospital notification procedure improves stroke outcome by shortening onset to needle time in Chinese urban area. Aging Dis 2018;9:426-434. 